\title{
Maximum Area with Minkowski Measures of Perimeter
}

\author{
Gilbert Strang* \\ Massachusetts Institute of Technology \\ gs@math.mit.edu math.mit.edu/ gs
}

\begin{abstract}
The oldest competition for an optimal shape (area-maximizing) was won by the circle. But if the fixed perimeter is measured by the line integral of $|d x|+|d y|$, a square would win. Or if the boundary integral of $\max (|d x|,|d y|)$ is given, a diamond has maximum area. For any norm in $\mathbf{R}^{2}$, we show that when the integral of $\|(d x, d y)\|$ around the boundary is prescribed, the area inside is maximized by a ball in the dual norm (rotated by $\pi / 2$ ).

This "isoperimetrix" was found by Busemann. For polyhedra it was described by Wulff in the theory of crystals. In our approach, the EulerLagrange equation for the support function of $S$ has a particularly nice form. This has application to computing minimum cuts and maximum flows in a plane domain.
\end{abstract}

\section{Introduction}

We can't really claim that Queen Dido of Carthage was a mathematician. But according to Virgil [56] she guessed the correct solution to an isoperimetric problem. Since the area to be maximized was partly bounded by water, she even went beyond the single constraint "perimeter $=L$ " that leads to a circle. The queen's solution was a semicircle perpendicular to the water (because the perimeter along that flat side did not count).

The authenticity of this example might be questioned, but it illustrates two themes of this small paper:

1. The definition of perimeter is crucial

2. A constraint $S \subset \Omega$ will affect the optimal shape $S$.

For the perimeter, part of the question is whether to count a boundary piece where $\partial S$ meets $\partial \Omega$. (Dido didn't and we will.) But answering a different question is the main purpose of this note. Suppose the measure of arclength is changed from

${ }^{*}$ This research was supported by the Singapore-MIT Alliance 
the $\ell^{2}$ norm $d s=\sqrt{(d x)^{2}+(d y)^{2}}$. We might use the $\ell^{1}$ norm $d s=|d x|+|d y|$ or any other norm on $\mathbf{R}^{2}$. Then the perimeter constraint $\int\left\|s^{\prime}(t)\right\| d t=L$ is changed, and a new shape $S$ provides maximum area.

We prove that the optimal $S$ is a rotated ball $\left\|(x, y)^{\perp}\right\|_{D} \leq R$, in the norm dual to the norm that defines arclength. Since $\ell^{\infty}$ is dual to $\ell^{1}$, the square $\max (|x|,|y|) \leq L / 8$ provides maximum area when the perimeter is $\int|d x|+|d y|=L$. The diamond $|x|+|y| \leq L / 4$ (a ball in $\ell^{1}$ ) is optimal when the perimeter is measured by $\int \max (|d x|,|d y|)=L$. Since $\ell^{2}$ is dual to itself, the classical isoperimetric problem produces a circle.

All these optimal shapes are known! Section 4 gives a brief history of this isoperimetric problem in the Minkowski plane - maximizing area when the perimeter is specified in a prescribed norm. The "isoperimetrix" $S$ was discovered by Busemann [10], applying the Brunn-Minkowski inequality [23]. For polyhedra, the optimal shape was described much earlier in Wulff's theory of crystals. Many authors have contributed, and our purpose is to give a direct and elementary argument using the calculus of variations.

Our approach computes perimeter and area from the support function $p(\phi)$ of $S$. In Figure $1, p(\phi)$ is the distance from $(0,0)$ to the tangent line normal to the direction $\phi$. The area is $\frac{1}{2} \int\left(p^{2}-p^{\prime 2}\right) d \phi$. The usual measure of perimeter $\left(\ell^{2}\right.$ norm) is $\int\left(p+p^{\prime \prime}\right) d \phi=L$. Minimizing area with this constraint quickly yields a circular shape $S: p=$ constant from equation (3.2).

That computation of the optimal shape generalizes directly to other norms, and the Euler-Lagrange equation (2.5) for the best $p(\phi)$ is linear.

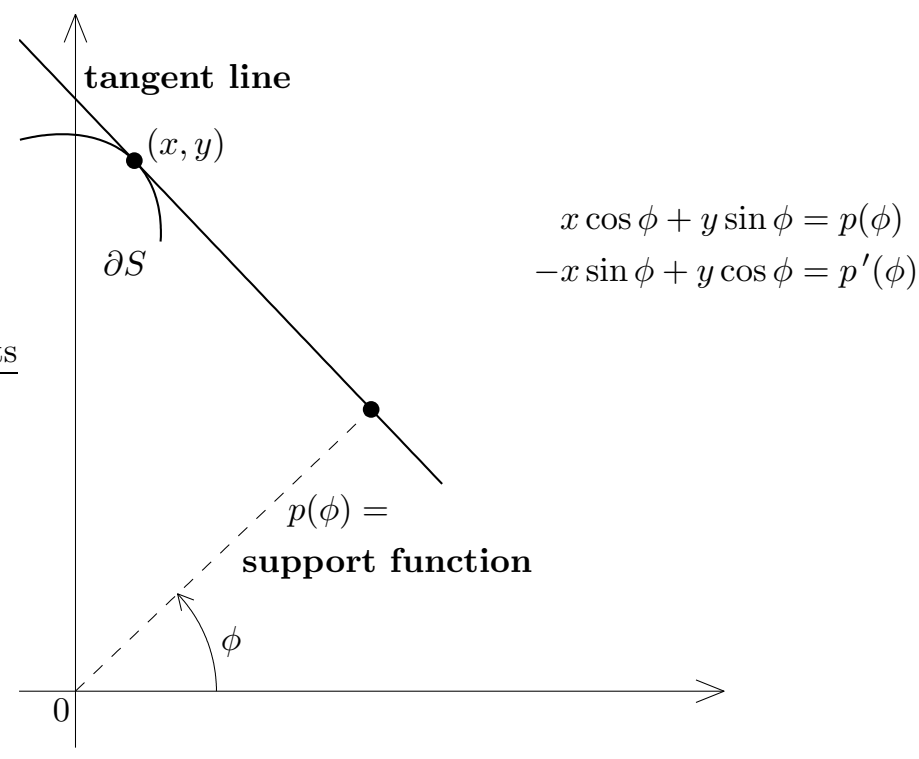

Figure 1: [Santaló, p. 2]. The line at distance $p$ is tangent to $\partial S$ at $(x, y)$. 
We met these new measures for the perimeter in identifying the minimum cut $\partial S$ in a plane domain $\Omega$ [51]:

$$
\text { Constrained isoperimetric problem } \quad \min \frac{|\partial S|}{|S|} \quad \text { for } S \subset \Omega .
$$

In the $\ell^{2}$ norm for $d s$, the minimum ratio is Cheeger's constant $h(\Omega)$. That number provides a lower bound $\lambda_{1} \geq h^{2} / 4$ for the eigenvalues of the Laplace-Dirichlet operator on $\Omega[13,25]$. The number $h$ is also (by duality) the largest value $\lambda$ such that $\operatorname{div} v=\lambda$ has a solution with $v_{1}^{2}+v_{2}^{2} \leq 1$ in $\Omega$.

That max flow-min cut theorem [51] for flows in a plane domain $\Omega$ holds for any norm of the velocity vector $v(x, y)$. This application of duality is remarkable for the fact that the minimum cut $\partial S$ may be easily determined from (1.1), while the flow vector $v(x, y)$ that fills the cut to capacity has only recently been approximated [37]. No analytic expression for $v$ has been discovered [50, 52].

We end with these applications after finding the Euler-Lagrange equations for maximum area (with perimeter $=L$ ).

After this paper was completed, we learned of new applications [28, 29, 30, 32] to landslide modeling (and blocking sets and the 1-Laplacian).

\section{A rotated dual ball is isoperimetrically optimal}

To start, the only constraint $\int\|(d x, d y)\|=L$ is on the perimeter of $S$. The problem is to maximize the area. Classical arguments show that the optimal shape $S$ is convex. We will compute its perimeter and area using the support function $p(\phi)$ the distance to the support line - rather than the position function $r(\theta)$.

The two equations in Figure 1 express the fact that $\partial S$ is the envelope of its tangent lines. Santaló [47] solves those equations for the point of tangency:

$$
x=p \cos \phi-p^{\prime} \sin \phi, \quad y=p \sin \phi+p^{\prime} \cos \phi .
$$

Differentiating these equations,

$$
d x=-\left(p+p^{\prime \prime}\right) \sin \phi d \phi, \quad d y=\left(p+p^{\prime \prime}\right) \cos \phi d \phi .
$$

The convexity of $S$ is equivalent to $p+p^{\prime \prime} \geq 0$. Then the perimeter of $S$ can be computed directly from $p(\phi)$ and the norm $N(\phi)$ of the unit tangent vector $(-\sin \phi, \cos \phi)$ :

$$
\text { Perimeter of } \boldsymbol{S}=\int_{0}^{2 \pi}\|(d x, d y)\|=\int_{0}^{2 \pi}\left(p+p^{\prime \prime}\right) N(\phi) d \phi .
$$

Since $N(\phi)=1$ in the $\ell^{2}$ norm and $p(\phi)$ is periodic, the usual measure of perimeter has the particularly nice form $\int p d \phi$.

The Euclidean area of $S$ is also given by a line integral involving $p(\phi)$. Santaló notes that if $S$ is decomposed into triangles of height $p(\phi)$ and base $d s=\left(p+p^{\prime \prime}\right) d \phi$ 
(vertex at the origin, Euclidean length along $\partial S$ ), then

$$
\text { Area of } S=\frac{1}{2} \int_{0}^{2 \pi} p\left(p+p^{\prime \prime}\right) d \phi=\frac{1}{2} \int_{0}^{2 \pi}\left(p^{2}-p^{\prime 2}\right) d \phi
$$

The last step was integration by parts of $p p^{\prime \prime}$, using the periodicity $p p^{\prime}(2 \pi)=$ $p p^{\prime}(0)$. A non-smooth support function (where $\partial S$ contains a line segment) can be approximated by a smoother $p(\phi) \in C^{2}$, to justify $(2.3)$ and $(2.4)$.

Those formulas produce a beautifully simple restatement of the isoperimetric problem:

$$
\text { Maximize } \frac{1}{2} \int_{0}^{2 \pi}\left(p^{2}-p^{\prime 2}\right) d \phi \text { subject to } \int_{0}^{2 \pi}\left(p+p^{\prime \prime}\right) N(\phi) d \phi=L .
$$

A Lagrange multiplier $\lambda$ builds the perimeter constraint into the functional $F(p, \lambda)=$ area $-\lambda$ (perimeter $-L)$. The calculus of variations sets the first variation of $F$ to zero. Equation (4.1) will recall this step, and here we go directly to the unusually neat form of the optimality condition:

$$
\text { Euler-Lagrange } \quad p(\phi)+p^{\prime \prime}(\phi)-\lambda\left[N(\phi)+N^{\prime \prime}(\phi)\right]=0 .
$$

The equation is linear in $p(\phi)$, not in the position variable $r(\theta)$. A particular solution to $(2.5)$ is $p(\phi)=\lambda N(\phi)$, and the complete solution includes arbitrary constants $A$ and $B$ :

$$
p(\phi)=\lambda N(\phi)+A \cos \phi+B \sin \phi .
$$

Centering $S$ horizontally and vertically, so that $p(0)=p(\pi)$ and $p(\pi / 2)=p(3 \pi / 2)$, achieves $A=0$ and $B=0$. Then the support function of the optimal shape is $\lambda N(\phi)$ :

$$
\text { Optimal shape } S \quad p(\phi)=\lambda N(\phi)=\lambda\|(-\sin \phi, \cos \phi)\| .
$$

This shape will be seen as a ball in the dual norm, scaled by $\lambda$ and rotated by $\pi / 2$.

\section{The classical isoperimetric problem}

The classical problem uses the $\ell^{2}$ norm, with $N(\phi)=\|(-\sin \phi, \cos \phi)\|=1$. We repeat the steps in detail for this special case, to emphasize how the isoperimetric problem is simplified by using the support function $p(\phi)$ :

$$
\text { Maximize } \frac{1}{2} \int_{0}^{2 \pi}\left(p^{2}-p^{\prime 2}\right) d \phi \quad \text { subject to } \int p d \phi=L .
$$

Suppose an optimal $p(\phi)$ is perturbed by $q(\phi)$. Then $\int q d \phi=0$ to maintain the perimeter constraint $\int(p+q) d \phi=L$. The new area is half of

$$
\int_{0}^{2 \pi}\left[(p+q)^{2}-\left(p^{\prime}+q^{\prime}\right)^{2}\right] d \phi=\int\left(p^{2}-p^{\prime 2}\right) d \phi-2 \int\left(p q-p^{\prime} q^{\prime}\right) d \phi+\int\left(q^{2}-q^{\prime 2}\right) d \phi .
$$


To ensure that $p(\phi)$ is maximizing, we need the first-order condition $\int\left(p q-p^{\prime} q^{\prime}\right) d \phi=$ 0 whenever $\int q d \phi=0$. We also need $\int\left(q^{2}-q^{\prime 2}\right) d \phi \leq 0$ for all $q$.

The first requirement, after integration by parts, produces the Euler-Lagrange equation $p+p^{\prime \prime}=$ constant:

$$
\int\left(p q-p^{\prime} q^{\prime}\right) d \phi=\int\left(p+p^{\prime \prime}\right) q d \phi=0 \quad \text { when } \quad \int q d \phi=0 \quad \text { needs } \quad p+p^{\prime \prime}=\lambda .
$$

This is the argument that becomes automatic with the introduction of a Lagrange multiplier. If $(u, q)=0$ implies $(v, q)=0$, then $u=\lambda v$. Here $p+p^{\prime \prime}=\lambda$ is equation (2.5) with $N=1$. The solution $p=\lambda$ produces a circle. The circle can be translated as in (2.6) by including in $p$ the homogeneous solution $A \cos \phi+B \sin \phi$.

The second requirement $\int q^{2} \leq \int q^{\prime 2}$ is exactly Wirtinger's inequality for any periodic $q(\phi)=\sum a_{n} e^{i n \phi}$, when we know that $a_{0}=\int q d \phi / 2 \pi=0$ :

$$
\text { Wirtinger } \quad \int_{0}^{2 \pi}\left(q^{2}-q^{\prime 2}\right) d \phi=2 \pi\left[\sum\left|a_{n}\right|^{2}-\sum n^{2}\left|a_{n}\right|^{2}\right] \leq 0 \text {. }
$$

This is an equality when the only nonzero Fourier coefficients have $n^{2}=1$. That allows the arbitrary $q(\phi)=A \cos \phi+B \sin \phi$ that we met in (2.6). If any nonzero coefficient of $q(\phi)$ has $n^{2}>1$, then (3.4) is a strict inequality. In that case the perturbation $q(\phi)$ of the optimal $p(\phi)=\lambda$ will decrease the area in (3.2).

The set $S$ with $p(\phi)=$ constant is a circle, the isoperimetric shape that was conjectured by the Greeks. Satisfactory proofs were found much later! For guides to the fascinating history of this problem see $[7,42,45]$. A short analytic proof was given by Lax [35].

Returning to more general norms, with the factor $N(\phi)=\|(-\sin \phi, \cos \phi)\|$ in the perimeter integral, the same steps (3.2)-(3.3) lead to the Euler-Lagrange equation. Now $\int\left(p+p^{\prime \prime}\right) q=0$ whenever $\int\left(N+N^{\prime \prime}\right) q=0$, so that $p+p^{\prime \prime}$ is proportional to $N+N^{\prime \prime}$. The solution is $p=\lambda N$.

We note a small twist to the usual proof that $S$ must be convex. If not convex, there will be a line not crossed by $S$ but tangent at two points. Normally we can move a region (between the line and $\partial S$ ) across that line. The mirror image leads to increased area with the same perimeter. With $N(\phi)$ included in the perimeter, we have to reverse the direction of that piece of $\partial S$, for the move to maintain a fixed perimeter.

Dacorogna and Pfister [15, 16] have extended Wirtinger's inequality (3.4) to this case of more general norms. Their analysis uses a parametric form $(x(s), y(s))$ for the boundary $\partial S$, and gives the proof in full detail.

The area-maximizing shape $S$ has $p(\phi)$ given by $\lambda N(\phi)$. By definition, the unit ball $\|(x, y)\|_{D} \leq 1$ in the dual norm has the support function $\|(\cos \phi, \sin \phi)\|$. Therefore $S$ has the same shape as that ball, after it is rescaled by $\lambda$ and rotated by $\pi / 2$. Figure 2 shows a particular unit ball and its dual.

With $p(\phi)=\lambda N(\phi)$, the ratio of perimeter to area is $2 / \lambda$ :

$$
\frac{\int\left(p+p^{\prime \prime}\right) N d \phi}{\int\left(p+p^{\prime \prime}\right) p d \phi / 2}=\frac{\lambda \int\left(N+N^{\prime \prime}\right) N d \phi}{\lambda^{2} \int\left(N+N^{\prime \prime}\right) N d \phi / 2}=\frac{2}{\lambda} .
$$




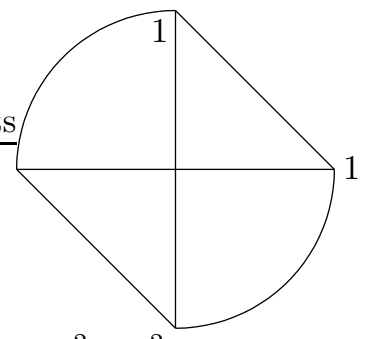

$\|(u, v)\|=\left\{\begin{array}{l}u+v \\ \sqrt{u^{2}+v^{2}}\end{array}\right.$

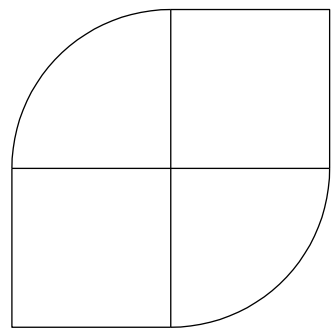

$\|(x, y)\|_{D}=1$

$N$-perimeter 8

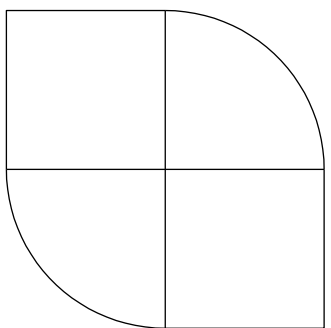

$\left\|(x, y)^{\perp}\right\|_{D}=1$

$N$-perimeter $4+\pi$

Figure 2: Dual unit balls have $|x u+y v| \leq 1$. Rotated ball has least perimeter.

The Lagrange multiplier $\lambda$ is determined by the value $L$ of the perimeter:

$$
L=\int_{0}^{2 \pi}\left(p+p^{\prime \prime}\right) N d \phi=\lambda \int_{0}^{2 \pi}\left(N+N^{\prime \prime}\right) N d \phi=\lambda \int_{0}^{2 \pi}\left(N^{2}-N^{\prime 2}\right) d \phi .
$$

\section{The isoperimetrix}

When those paragraphs were written, using the calculus of variations to identify the optimal shape $S$, I did not know whether this problem (in an arbitrary norm) was new. It is definitely not new! Sixty years ago Busemann [10] asked exactly the same question, and gave $S$ the name isoperimetrix. This construction became a key result in convex geometry [55], following Minkowski's fundamental idea that every norm on $\mathbf{R}^{n}$ defines a length and area and volume.

One family of inequalities is at the center of that subject. The Brunn-Minkowski inequality states that the $n$th root of the volume is a concave function on the set of convex bodies. When Busemann reduced the isoperimetric problem to that inequality, his proof was complete. He did not need an Euler-Lagrange equation for the boundary curve $\partial S$, and we have not found this equation for $p(\phi)$ in the literature.

Geometry leads to Minkowski's concept of a mixed volume $V\left(K_{1}, K_{2}\right)$, which is the coefficient of $a b$ in the volume of a convex combination $a K_{1}+b K_{2}$. The 2-dimensional inequality that Brunn proved becomes $V^{2}\left(K_{1}, K_{2}\right) \geq V\left(K_{1}\right) V\left(K_{2}\right)$. In fact Gromov has pointed to a mention of the isoperimetrix in Brunn's 1887 inaugural dissertation. The inequalities that Minkowski uncovered made it possible for Busemann to extend his isoperimetrix to $\mathbf{R}^{n}$ [11].

To an outsider, the Euler-Lagrange equation is more familiar. Integration by parts quickly produces the first variation of $F=\int f\left(p, p^{\prime}, p^{\prime \prime}\right) d \phi$ :

$$
\text { Euler-Lagrange } \quad \frac{\partial f}{\partial p}-\frac{d}{d \phi}\left(\frac{\partial f}{\partial p^{\prime}}\right)+\frac{d^{2}}{d \phi^{2}}\left(\frac{\partial f}{\partial p^{\prime \prime}}\right)=0 .
$$


Applied to $f=p\left(p+p^{\prime \prime}\right) / 2-\lambda\left(p+p^{\prime \prime}\right) N$, this gives equation (2.5) and the solution $p(\phi)=\lambda N(\phi)$. Euclidean geometry has $N(\phi)=1$, and then $p(\phi)=\lambda$ produces a circle. We apologize to ask any reader to choose between geometry and analysis.

One beautiful sidelight on the isoperimetrix appears in [57]. Wallen noticed that Kepler's Law of equal areas swept out in equal times holds for a planet that travels along the optimal $\partial S$ with constant Minkowski speed. In our notation, this is the statement that $p\left(p+p^{\prime \prime}\right) / 2$ is in fixed proportion to $\left(p+p^{\prime \prime}\right) N$ because $p=\lambda N$.

We also owe to Wallen [58] a new appreciation of Wirtinger's inequality in equation (3.4). This can yield the Brunn-Minkowski inequality [8, p.114] and Bonnesen's refinement $[20,57]$ and the classical isoperimetric inequality $[15,16]$. Every argument for a minimum or maximum needs convexity or concavity at some point. Wirtinger's inequality provides that here, by confirming that the last term in (3.2) decreases the area.

When the unit balls are polyhedra (as for $\ell^{1}$ and $\ell^{\infty}$ ) the vertex directions in one ball are normal to the flat sides of the other ball. The isoperimetrix in this polyhedral case is the Wulff shape [60] in the theory of crystals. The energy is given by the (normed) surface area of the faces, and minimized.

The isoperimetrix has a long and important history in geometric measure theory. The existence of a minimizing $S$ and its uniqueness and form have been established for increasingly general domains and integrands [1, 9, 21, 41, 53]. Morgan outlines a history of this effort [40, see also 37, 44] that is fascinatingly independent of convex geometry. It was Gromov who connected both approaches to the work of Levy [36] by a new proof of the Brunn-Minkowski inequality [5, 26]. That proof rests on the divergence theorem, which was applied to the symmetrization map by Knothe [33].

Certainly this deeper analysis admits candidate shapes for which the first variation in (4.1) is not immediately defined. The proofs are far from elementary. The reader may wish to regard the Euler-Lagrange equation as decisive only when $\partial S$ is piecewise smooth.

\section{The constraint $S \subset \Omega$}

Suppose we remove the constraint of a fixed perimeter, and require instead that $S$ must be a subset of a given plane domain $\Omega$. The ratio of perimeter to area will be minimized by a set that touches the boundary $\partial \Omega$. (Rescaling $S$ by a factor $a>1$ will decrease the ratio, so the optimal $S$ cannot lie strictly inside $\Omega$.) In fact $\partial S$ is tangent to $\partial \Omega$ at points where they meet.

This is the opposite of Queen Dido's semicircle, which was perpendicular to $\partial \Omega$ because the piece $\partial \Omega \cap \partial S$ was not counted in the perimeter of $S$. In both problems, $S$ has the shape of an isoperimetrix in the interior of $\Omega$. (In the language of optimization, the constraint $S \subset \Omega$ is not active inside $\Omega$.) This section concentrates on calculating $S$ and understanding it as a minimum cut, without proofs.

For an explicit calculation we take $\Omega$ to be a unit square. A typical isoperimetrix is drawn in Figure 3, and its four pieces can fit (tangent to $\partial \Omega$ ) into the corners of the square. For simplicity we suppose that $(1,0)$ and $(0,1)$ lie on the dual unit ball, so the flat pieces $\partial S \cap \partial \Omega$ are measured by their length. Our problem is to 
normed perimeter $\alpha L$
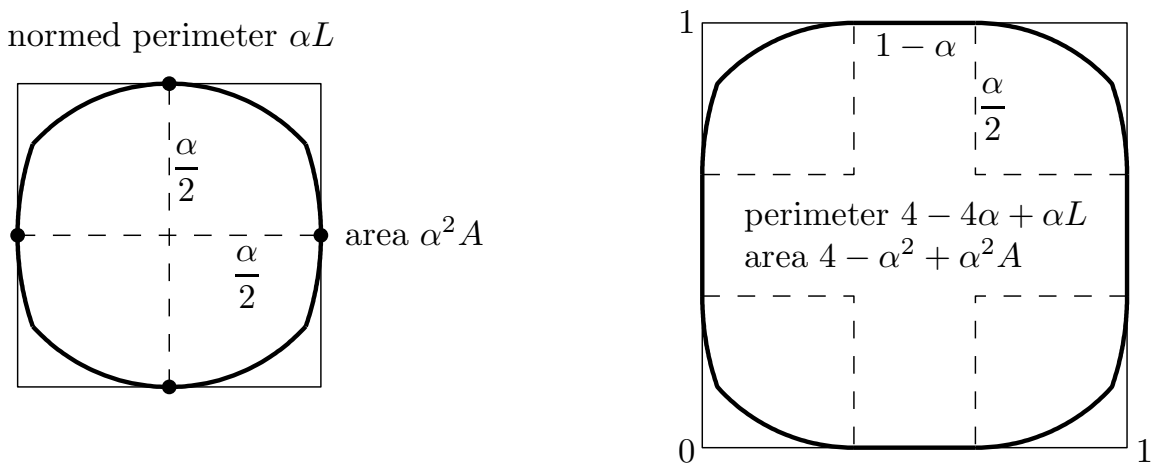

Figure 3: Four pieces of $\alpha$ (isoperimetrix) yield the constrained isoperimetrix.

determine the optimal scaling factor $\alpha \leq 1$ to minimize the ratio of perimeter to area:

$$
\frac{\text { Perimeter }}{\text { Area }}=\frac{4-4 \alpha+\alpha L}{1-\alpha^{2}+\alpha^{2} A} \text {. }
$$

The derivative of this ratio is zero when

$$
\left(1-\alpha^{2}+\alpha^{2} A\right)(L-4)=(4-4 \alpha+\alpha L) 2 \alpha(A-1) .
$$

That quadratic equation can be written as

$$
\alpha^{2} C D-8 \alpha D+C=0 \quad \text { with } \quad C=4-L \quad \text { and } \quad D=1-A .
$$

Comparing (5.1) and (5.2), the minimum ratio will be $C / 2 \alpha D$. It is simpler to solve (5.3) for the reciprocal $\beta=1 / \alpha \geq 1$ :

$$
\beta=\frac{4 D+\sqrt{(4 D)^{2}-C^{2} D}}{C}
$$

Then the optimum ratio is

$$
\frac{\text { Perimeter }}{\text { Area }}=\frac{\beta C}{2 D}=2+\sqrt{4-\frac{C^{2}}{4 D}} .
$$

In the $\ell^{2}$ norm, the isoperimetrix inside the unit square is a circle of perimeter $\pi$ and area $\frac{\pi}{4}$. Cheeger's constant with four quarter circles and boundary segments is then $2+\sqrt{\pi}$ :

$$
\frac{C^{2}}{4 D}=\frac{(4-\pi)^{2}}{4-\pi}=4-\pi \quad \text { and } \quad \frac{\text { Perimeter }}{\text { Area }}=2+\sqrt{\pi} .
$$




\section{Max Flow-Min Cut Theorem}

This final section describes the application which led us to the constraint $S \subset \Omega$ and to the minimum problem for the ratio perimeter/area. The starting point is a maximum problem for flow through the domain $\Omega$. We begin with the specific question that is answered by the number $2+\sqrt{\pi}$ (the minimum ratio computed just above).

Maximum Flow Problem Find the vector $v=\left(v_{1}(x, y), v_{2}(x, y)\right)$ that has the largest possible divergence $\lambda$, constant in the unit square $\Omega$, with the constraint $v_{1}^{2}+v_{2}^{2} \leq 1$ in $\Omega$ :

Maximize $\lambda$ such that $\operatorname{div} v=\frac{\partial v_{1}}{\partial x}+\frac{\partial v_{2}}{\partial y}=\lambda \quad$ with $\quad\|v\| \leq 1 \quad$ at all $x, y$ in $\Omega$.

This can be seen as a model of physical phenomena that are linear up to a pointbut that point $\|v\|=1$ cannot be passed. If we twist a long plastic cylinder with cross-section $\Omega$, then at some level of torsion, the stresses $v_{1}$ and $v_{2}$ cannot be sustained. The equilibrium $\operatorname{div} v=\lambda$ becomes incompatible (for any distribution of stresses) with $\|v\| \leq 1$. The cylinder reaches a yield limit and collapses. The collapse will occur along a "minimum cut" where perimeter/area $=2+\sqrt{\pi}$.

The key is the divergence theorem applied to subsets $S \subset \Omega$.

$$
\iint_{S} \operatorname{div} v d x d y=\int_{\partial S} v \cdot n d s \quad \text { so that } \quad \lambda \text { times area }|S| \leq \text { perimeter }|\partial S| .
$$

That inequality came from $|v \cdot n| \leq 1$. The continuous max flow-min cut theorem (established by duality theory in [51]) leads to the optimality condition: equality will hold on the extremal set $S$, where $|\partial S| /|S|$ is a minimum. At that moment, equation (6.2) assures that $v=n$ along the cut. But we don't know the vector field $v(x, y)$ that achieves $\lambda_{\max }=2+\sqrt{\pi}$. This maximum flow has been approximately computed by discrete quadratic programming [37].

For the discrete problem of maximum flow through a network, the minimum cut separates a subset of nodes including the "source" from the complementary subset including the "sink." All edges across the cut are filled to capacity. A finite algorithm increases the flow up to this maximum, and at that point the minimal cut (the bottleneck in the flow) is identified. This source-sink framework corresponds to $\operatorname{div} v=0$ in the interior of $\Omega$, given in the discrete case by Kirchhoff's Current Law that flow into a node equals flow out. Our specific problem has source term $\lambda$ inside $\Omega$ rather than on $\partial \Omega$, and the general case [51] has both interior sources $\lambda F(x, y)$ and boundary sources $\lambda f(x, y)$.

A more widely studied example is the Monge-Kantorovich continuous analog [18, 22 ] of the transportation problem on a discrete network. Again the applications are remarkable but the theory is not entirely constructive. We are asked to transport a mass from one density distribution to another, with minimum work. (For Monge, those were earth defenses demanded by Napoleon.) The key rule is that the shortest paths of mass movement will never cross. 
The maximum flow problem is finding unexpected uses in medical image segmentation [3] and in stereo reconstruction. We refer to our survey [52] for the essential role of the coarea formula in converting the dual to maximum flow into the constrained isoperimetric problem that directly identifies the minimum cut $\partial S$ :

Maximum flow Maximize $\lambda$ such that $\operatorname{div} v=\lambda$ and $\|v\| \leq 1$ in $\Omega$

Formal dual Minimize $\iint_{\Omega}\|\operatorname{grad} u\| d x d y$ with $\iint u d x d y=1$

Minimum cut $\quad$ Minimize (perimeter of $S$ )/(area of $S$ ) for $S \subset \Omega$.

The coarea formula expresses $\iint\|\operatorname{grad} u\| d x d y$ as an integral over the perimeters of level sets $S_{t}=\{u(x, y) \leq t\}$. This identifies the extreme points of the unit ball in the bounded variation norm $\|u\|_{\mathrm{BV}}=\iint\|\operatorname{grad} u\| d x d y$. Those extreme points are multiples of characteristic functions $\chi_{S}$ (equal to 1 on $S$ and elsewhere zero, with $\|\chi\|_{\mathrm{BV}}=$ perimeter of $S$ ). When the search for a minimum in the formal dual is restricted to $u=\chi_{S} /($ area of $S)$, we have the constrained isoperimetric problem of minimizing $|\partial S| /|S|$ for $S \subset \Omega$.

Finally, other norms on $\mathbf{R}^{2}$ will appear as soon as we change the pointwise constraint on $v(x, y)$ in the original maximum problem:

If the constraint becomes $\|v\| \leq 1$ for a different norm, then the dual of that norm appears in $\iint\|\operatorname{grad} u\|_{\mathrm{D}} d x d y$ and in the coarea formula and in the perimeter of $S$.

Any part of $\partial S$ inside $\partial \Omega$ will have the form of the Busemann-Wulff isoperimetrix. Our contribution was to find the support function of that set as $p(\phi)=\lambda N(\phi)$, directly from the Euler-Lagrange equation $p+p^{\prime \prime}=\lambda\left(N+N^{\prime \prime}\right)$.

\section{References}

[1] F. Almgren, Optimal isoperimetric inequalities, Indiana U. Math. J. 35 (1986) 451-547.

[2] J. C. Alvarez Paiva and A. Thompson, On the perimeter and area of the unit disk, American Mathematical Monthly 112 (2005) 141-154.

[3] B. Appleton and H. Talbot, Globally minimal surfaces by continuous Maximal Flows, IEEE Transactions on Pattern Analysis and Machine Intelligence 28 (2006) 106-118.

[4] R. V. Benson, Euclidean Geometry and Convexity, McGraw-Hill, 1966.

[5] M. Berger, Geometry II, Springer, 1987.

[6] W. Blaschke, Kreis und Kugel, Veit, Leipzig, 1916; Chelsea, 1949.

[7] V. Blåsjö, The isoperimetric problem, American Mathematical Monthly 112 (2005) 526-566. 
[8] T. Bonnesen and W. Fenchel, Theory of Convex Bodies, BCS Associates, 1987, translation by L. Boron, C. Christenson and B. Smith of Theorie der convexen Körper, Springer (1934).

[9] J. E. Brothers and F. Morgan, The isoperimetric theorem for general integrands, Michigan Math. J. 41 (1994) 419-431.

[10] H. Busemann, The isoperimetric problem in the Minkowski plane, American J. Math. 69 (1947) 863-871.

[11] H. Busemann, The isoperimetric problem for Minkowski area, Amer. J. Math. 71 (1949) 743-762.

[12] G. D. Chakerian, The isoperimetric problem in the Minkowski plane, Amer. Math. Monthly 67 (1960) 1002-1004.

[13] J. Cheeger and D. G. Ebin, Comparison Theorems in Riemannian Geometry, North-Holland, 1975.

[14] R. Courant and H. Robbins, What is Mathematics?, Oxford University Press, 1960.

[15] B. Dacorogna, Introduction to the Calculus of Variations, Imperial College Press, World Scientific, 2004.

[16] B. Dacorogna and C. E. Pfister, Wulff theorem and best constant in Sobolev inequality, J. Math. Pures Appl. 71 (1992) 97-118.

[17] E. DeGiorgi, Sulla proprietà isoperimetrica dell'ipersfera, nella classe degli insiemi aventi frontiera orientata di misura finita, Atti Accad. Naz. Lincei Mem. Cl. Sci. Fis. Mat. Nat. 5 (1958) 33-44.

[18] L. C. Evans, Survey of applications of PDE methods to Monge-Kantorovich mass transfer problems, www.math.berkeley.edu/ evans.

[19] Ky Fan, O. Taussky, and J. Todd, Discrete analogs of inequalities of Wirtinger, Monatsh. Math. 59 (1955), 73-90.

[20] H. Flanders, A proof of Minkowski's inequality for convex curves, American Mathematical Monthly 75 (1968) 581-593.

[21] I. Fonseca and S. Müller, A uniqueness proof for the Wulff theorem, Proc. Roy. Soc. Edinburgh Sect. A 119 (1991) 125-136.

[22] W. Gangbo and R. McCann, Optimal maps in Monge's mass transport problem, C.R. Acad. Sci. Paris. Ser. I. Math. 325 (1995) 1653-1658.

[23] R. J. Gardner, The Brunn-Minkowski inequality, Bulletin American Math Soc. 39 (2002) 355-405.

[24] S. Golab, Sur la longueur de l'indicatrice dans la géometrie plane de Minkowski, Colloq. Math. 15 (1966) 141-144. 
[25] D. Grieser, The first eigenvalue of the Laplacian, isoperimetric constants, and the max flow min cut theorem, Archiv der Mathematik 87 (2006) 75-85.

[26] M. Gromov, Isoperimetric inequalities in Riemannian manifolds, Appendix I in Asymptotic Theory of Finite Dimensional Normed Spaces, V. D. Milman and G. Schechtman, Springer Lecture Notes in Mathematics 1200 (1986) 114-129.

[27] B. Grünbaum, The perimeter of Minkowski unit discs, Colloq. Math. 13 (1966) $135-139$.

[28] R. Hassani, I. R. Ionescu, T. Lachand-Robert, Shape optimization and supremal functionals in landslides modeling, Applied Mathematics and Optimization 52 (2005) 349-364.

[29] P. Hild, I. R. Ionescu, T. Lachand-Robert, I. Rosca, The blocking property of an inhomogeneous Bingham fluid. Applications to landslides, Mathematical Modelling and Numerical Analysis 36 (2002) 1013-1026.

[30] I. R. Ionescu and T. Lachand-Robert, Generalized Cheeger's sets related to landslides, Calculus of Variations and PDE 23 (2005) 227-249.

[31] F. John, Extremum problems with inequalities as subsidiary conditions, in Studies and Essays Presented to R. Courant on His 60th Birthday (eds. K. O. Friedrichs, O. Neugebauer and J. J. Stoker), Interscience (1948) 187-204.

[32] B. Kawohl and V. Fridman, Isoperimetric estimates for the first eigenvalue of the $p$-Laplace operator and the Cheeger constant, Comm. Mat. Univ. Carolinae 44 (2003) 659-667.

[33] H. Knothe, Contributions to the theory of convex bodies, Michigan Math. J. 4 (1957) 39-52.

[34] G. Lawlor, A new area maximization proof for the circle, Mathematical Intelligencer 20 (1999) 29-30.

[35] P. Lax, A short path to the shortest path, Amer. Math. Monthly 102 (1995) $158-159$.

[36] P. Levy, Problèmes Concrets d'Analyse Fonctionnelle, Gauthier-Villars, 1951.

[37] R. Lippert, Discrete approximations to continuum optimal flow problems, Studies in Applied Mathematics, to appear.

[38] L. A. Lyusternik, Convex Figures and Polyhedra, Heath, 1966.

[39] H. Martini, K. J. Swanepoel, and G. Weiss, The geometry of Minkowski spaces - a survey, Expositiones Mathematicae, Part I, 19 (2001) 97-142, and Part II, 22 (2004) 93-144.

[40] F. Morgan, Riemannian Geometry: A Beginner's Guide, Jones and Bartlett, 1993; Second edition, AK Peters, 1998. 
[41] F. Morgan, Planar Wulff shape is unique equilibrium, Proc. Amer. Math. Soc. 133 (2005) 809-813.

[42] R. Osserman, The isoperimetric inequality, Bull. Amer. Math. Soc. 84 (1978) $1182-1238$.

[43] Pappus of Alexandria, Book 5: La Collection Mathematique, Desclee, de Brouwer, 1933.

[44] G. Pisier, The Volume of Convex Bodies and Banach Space Geometry, Cambridge University Press, 1989.

[45] T. I. Porter, A history of the classical isoperimetric problem, in Contributions to the calculus of variations (1931-1932), edited by G. A. Bliss and L. M. Graves, University of Chicago Press, 1933.

[46] W. Rzymowski and A. Stachura, Curves bounding maximal area, Nonlinear Analysis: Theory, Methods, and Applications 20 (1993) 1369-1372.

[47] L. A. Santaló, Integral Geometry and Geometric Probability, Addison-Wesley, 1976.

[48] J. J. Schäffer, The self-circumference of polar convex bodies, Arch. Math. 24 (1973) 87-90.

[49] R. Schneider, Convex Bodies: The Brunn-Minkowski Theory, Encyclopedia of Math. and Its Appl. 44, Cambridge University Press, 1993.

[50] G. Strang, $L^{1}$ and $L^{\infty}$ approximation of vector fields in the plane, Lecture Notes in Num. Appl. Anal. 5 (1982) 273-288.

[51] G. Strang, Maximal flow through a domain, Math. Programming 26 (1983) 123-143.

[52] G. Strang, Maximum flows and minimum cuts in the plane (2006), to appear in the Journal of Global Optimization.

[53] J. Taylor, Crystalline variational problems, Bull. Amer. Math. Soc. 84 (1978) $568-588$.

[54] A. A. Thompson and A. C. Thompson, The divergence theorem and the Laplacian in Minkowski space, Geom. Dedicata 63 (1996) 159-170.

[55] A. C. Thompson, Minkowski Geometry, Cambridge University Press, 1996.

[56] Virgil (Publius Vergilius Maro), Aeneid I, 365-368.

[57] L. J. Wallen, Kepler, the taxicab metric, and beyond: An isoperimetric primer, College Math. Journal 26 (1995) 178-190.

[58] L. J. Wallen, All the way with Wirtinger: A short proof of Bonnesen's inequality, American Mathematical Monthly 94 (1987) 440-442. 
[59] R. Webster, Convexity, Oxford University Press, Oxford, 1994.

[60] G. Wulff, Zur Frage der Geschwindigkeit des Wachsthums und der Auflösung der Krystallflachen, Zeitschrift für Krystallographie und Mineralogie 34 (1901) 449-530. 